\title{
Evidence for a "Pathogenic Triumvirate" in Congenital Hepatic Fibrosis in Autosomal Recessive Polycystic Kidney Disease
}

\author{
Lu Jiang, Pingping Fang, James L. Weemhoff, Udayan Apte, and Michele T. Pritchard \\ Department of Pharmacology, Toxicology and Therapeutics, University of Kansas Medical Center, 3901 Rainbow Blvd, \\ Kansas City, KS 66160, USA \\ Correspondence should be addressed to Michele T. Pritchard; mpritchard@kumc.edu
}

Received 30 June 2016; Revised 20 September 2016; Accepted 13 October 2016

Academic Editor: Guangcun Huang

Copyright (c) $2016 \mathrm{Lu}$ Jiang et al. This is an open access article distributed under the Creative Commons Attribution License, which permits unrestricted use, distribution, and reproduction in any medium, provided the original work is properly cited.

\begin{abstract}
Autosomal recessive polycystic kidney disease (ARPKD) is a severe monogenic disorder that occurs due to mutations in the PKHD1 gene. Congenital hepatic fibrosis (CHF) associated with ARPKD is characterized by the presence of hepatic cysts derived from dilated bile ducts and a robust, pericystic fibrosis. Cyst growth, due to cyst wall epithelial cell hyperproliferation and fluid secretion, is thought to be the driving force behind disease progression. Liver fibrosis is a wound healing response in which collagen accumulates in the liver due to an imbalance between extracellular matrix synthesis and degradation. Whereas both hyperproliferation and pericystic fibrosis are hallmarks of CHF/ARPKD, whether or not these two processes influence one another remains unclear. Additionally, recent studies demonstrate that inflammation is a common feature of CHF/ARPKD. Therefore, we propose a "pathogenic triumvirate" consisting of hyperproliferation of cyst wall growth, pericystic fibrosis, and inflammation which drives CHF/ARPKD progression. This review will summarize what is known regarding the mechanisms of cyst growth, fibrosis, and inflammation in CHF/ARPKD. Further, we will discuss the potential advantage of identifying a core pathogenic feature in $\mathrm{CHF} / \mathrm{ARPKD}$ to aid in the development of novel therapeutic approaches. If a core pathogenic feature does not exist, then developing multimodality therapeutic approaches to target each member of the "pathogenic triumvirate" individually may be a better strategy to manage this debilitating disease.
\end{abstract}

\section{Introduction}

Autosomal recessive polycystic kidney disease (ARPKD) is a rare genetic disorder that occurs in 1:20,000 live births. It develops in utero and is mainly diagnosed in pregnancy or in the immediate neonatal period. Among all affected patients, approximately $30 \%$ die shortly after birth, primarily of pulmonary insufficiency [1]. Patients who survive the neonatal period present with a broad spectrum of symptoms involving the kidneys, liver, and pancreas. Renal manifestations are characterized by the presence of cysts that are derived from dilated collecting ducts and distal tubules [2,3]. A significant portion of patients will progress to end stage renal disease either during the first decade or during adolescence [4]. A minority of patients develop pancreatic abnormalities consisting of cysts and fibrosis [5, 6]. All patients with ARPKD develop some degree of congenital hepatic fibrosis $(\mathrm{CHF})$, which, as the name would suggest, is present at birth. CHF is characterized by bile duct dilation resulting in eventual development of cysts and pericystic fibrosis in the liver $[7,8]$. Accompanying cyst growth and fibrosis, recent reports suggest that inflammation is also present and likely contributes to disease pathogenesis and/or progression [912]. Aside from management of symptoms and liver and/or kidney transplant, no effective pharmacologic therapies exist for CHF/ARPKD [13].

Although CHF is most commonly thought to be associated with ARPKD, there are several cases reported in autosomal dominant polycystic kidney disease (ADPKD). The patients with ADPKD showed hepatic cysts and fibrosis at birth, which is consistent with symptoms described in CHF/ARPKD [14]. Other ciliopathies in which $\mathrm{CHF}$ is found include Meckel-Gruber syndrome [15], renal-hepaticpancreatic dysplasia (an autosomal recessive disorder with renal dysplasia and pancreatic fibrosis) [16], and $\mathrm{COACH}$ syndrome (a subset of Joubert Syndrome Related Disorders, 
TABLE 1: Current rodent models of ARPKD.

\begin{tabular}{|c|c|c|c|c|c|}
\hline Model & Species & Liver phenotype & Kidney phenotype & Other phenotypes & Reference \\
\hline$\overline{\mathrm{PCK}}$ & Rat & Cysts and fibrosis & Cysts & Pancreatic cysts & [29] \\
\hline $\mathrm{BALB} / \mathrm{c}-c p k / c p k$ & Mouse & Cysts and fibrosis & Cysts & Pancreatic cysts and fibrosis & {$[33]$} \\
\hline $\mathrm{C} 57 \mathrm{BL} / 6 \mathrm{~J}-c p k / c p k$ & Mouse & No liver disease & Cysts & None & {$[34]$} \\
\hline Pkhd1 ${ }^{\text {del2/del2 }}$ & Mouse & Cysts and fibrosis & Cysts in female & Pancreatic cysts & {$[35]$} \\
\hline Pkhd1 $1_{\text {LSL(-)/LSL(-) }}$ & Mouse & Cysts and fibrosis & Cysts in female & Unknown & {$[36]$} \\
\hline Pkhdlexon40 & Mouse & Cysts and fibrosis & No kidney disease & Portal hypertension & [37] \\
\hline Pkhd1 $1^{\text {lacZ/lacZ }}$ & Mouse & Cysts and fibrosis & Cysts & Pancreatic and gall bladder cysts & [5] \\
\hline Pkhd $1^{\text {del4/del } 4}$ & Mouse & Cysts and fibrosis & No kidney disease & Pancreatic cysts, splenomegaly & {$[38]$} \\
\hline
\end{tabular}

an autosomal recessive multisystemic disorder with cerebellar vermis hyperplasia, ataxia, and mental retardation) [17].

\section{Gene Defects in ARPKD}

CHF/ARPKD is caused by mutations in the PKHD1 gene. PKHD1 extends over $470 \mathrm{~kb}$, includes a minimum of 86 exons, and encodes a 4,074-amino-acid protein called fibrocystin/polyductin. Fibrocystin is predicted to be a receptorlike protein that consists of a large glycosylated extracellular region, a single transmembrane domain, and a short cytoplasmic tail $[18,19]$. Fibrocystin is expressed in the primary cilia of epithelial cells. Immunohistochemistry studies suggest that fibrocystin is located in renal collecting ducts and loops of Henle, pancreatic epithelial ducts, and hepatic biliary ducts [20]. Different mutations in PKHD1 have been described in human ARPKD patients, including missense mutations, deletion/insertion mutations, and splicing mutations. Among all types of mutations found in PKHD1, about $45 \%$ of them are predicted to truncate fibrocystin [21]. Disease in patients carrying two truncating mutations is usually more severe, whereas patients bearing missense mutations exhibit a milder phenotype [22].

\section{Current Therapies for ARPKD}

There is currently no pharmacologic cure for CHF/ARPKD. Treatment mainly focuses on management of symptoms and includes therapies for cardiac hypertension, chronic liver/kidney disease, cholangitis, and portal hypertension [23]. Hypertension associated with chronic kidney disease occurs at the early stage of disease and is regulated by the renin-angiotensin system (RAS) [24]. Hypertension in ARPKD is treated empirically. Angiotensin converting enzyme (ACE) inhibitors and angiotensin II receptor blockers (ARBs) are considered the main treatment options in ARPKD patients $[8,25]$. If kidney failure occurs, patients undergo dialysis or kidney transplantation. CHF/ARPKD can be accompanied by recurrent cholangitis and cholangiocarcinoma. Although the occurrence of recurrent cholangitis and cholangiocarcinoma is relatively rare, liver transplantation is indicated to decrease mortality [26, 27]. Other therapeutic strategies include targeting components of the cAMP signaling pathway since cAMP levels are increased in cyst wall epithelial cells (CWECs) and drive CWEC proliferation. Octreotide and pasireotide, two somatostatin analogs, decrease proliferation of PCK rat CWEC in vitro and inhibit hepatorenal cyst growth in PCK rats in vivo by reducing CAMP levels. Consistently, clinical trials in patients with polycystic liver disease (PLD) and ADPKD showed that octreotide or lanreotide is well tolerated and decreases total liver volume by $4 \%-6 \%$ [13].

\section{Animal Models of ARPKD}

A number of rodent models of human ARPKD exist to study the mechanisms of disease and to test therapeutic strategies (Table 1). One of the best-characterized models is the polycystic kidney (PCK) rat, derived from SpragueDawley (SD) rats at Charles River, Inc. [28]. The PCK rat carries a spontaneous splicing mutation, IVS35-2A $\rightarrow \mathrm{T}$, in the rat $P k h d 1$ gene [19]. PCK rats bear hepatic and renal cysts and associated fibrosis, similar to human ARPKD [29]. The lifespan of a PCK rats is about 1.5 years, and they develop numerous cysts in kidneys and liver by one year of age [30]. In mice, the congenital polycystic kidney ( $c p k)$ mouse mimics human ARPKD. This mouse harbors a spontaneous mutation in $c p k$ gene, the gene that encodes a 145 -amino-acid protein termed cystin. Cystin is mainly located in the axoneme of the primary cilia found in the kidney proximal tubules and collecting ducts and in the cholangiocytes found in the liver $[31,32]$. cpk mice, on the BALB/c background, exhibit both renal and extrarenal manifestations associated with cystin mutations [33]. When on a C57Bl/6J background, $c p k$ mice do not have extrarenal pathology [34], limiting the utility of this model for those interested in studying CHF/ARPKD. In addition, $P k h d 1^{\text {del2/del2 }}$ mouse model, which lacks exon 2 of the mouse $P k h d 1$ gene, also reproduces the human ARPKD pathology. Female mice develop dilation of the renal proximal tubule and cysts by 3 months of age, whereas male mice are protected from renal cysts. Both genders develop hepatic cysts and fibrosis by 3 months as a result of biliary ductal plate malformation [35]. Another widely accepted murine model with $P k h d 1$ mutation was generated by Christopher Ward and colleagues. In this model, the Pkhdl gene was transcriptionally silenced by inserting a loxP flanked STOP (LSL) cassette into intron-2. Pkhd1 $1^{\mathrm{LSL}(-) / \operatorname{LSL}(-)}$ mice, both male and female, develop liver cysts and fibrosis at 3 months of age [36]. In addition, by disrupting exon 40, homozygous Pkhd1 mutant mice exhibit severe hepatic cysts and pericystic fibrosis in neonates due to biliary malformation in the embryo. However, the morphology and function in kidneys 
are not affected [37]. Another well-described murine model is the homozygous $P k h d l$ lacZ/lacZ mice. They are widely accepted as an ARPKD mouse model due to the presence of both hepatic and renal manifestations [5]. Pkhd1 del4/del4 mouse is described predominantly as a model for $\mathrm{CHF}$, since the kidneys are unaffected by the mutation [38]. Among all rodent models for ARPKD, the PCK rat is one of the few commercially available models to date, and the phenotypic resemblance to human ARPKD makes it an incredibly valuable resource for $\mathrm{CHF} / \mathrm{ARPKD}$ researchers.

\section{Mechanisms of Cystogenesis in ARPKD}

Although the mechanisms of cystogenesis are not well characterized in human ARPKD, a study using PCK rats suggested a possible link between cystogenesis and ciliary dysfunction [39]. Primary cilia, microtubule-based organelles, extend from the surface of eukaryotic cells. Primary cilia are nonmotile cilia containing a " $9+0$ " axoneme, and function as mechano-, osmo-, and chemosensors that deliver signals from the extracellular environment into the cell [40]. The abnormal primary cilia in PCK rat cholangiocytes may compromise their sensory organelle function in response to fluid secretion or fluid flow. Recent research suggests that primary cilia are also important components of multiple signaling pathways such as the hedgehog and PDGF-A signaling pathways [41, 42].

Hepatic cyst development in human ARPKD patient is characterized by abnormal remodeling of ductal plate from the double cell layer. Clinical manifestations include dilated bile ducts, an increased number of bile ducts, and abnormal branching [8]. Whether the hepatic cysts are disconnected from the biliary as they grow remains to be studied although it is the case in ADPKD patients [43]. Cyst development in PCK rat liver has been well-described by Dr. La Russo's group $[13,44,45]$. They found that (1) hepatic cysts are derived from bile duct segments due to ductal plate malformation during development and (2) most cysts become isolated from biliary tree by 6 months of age [39]. Along with cyst formation, the mechanisms of cyst expansion are proposed to be the result of the following: (1) cholangiocyte hyperproliferation, (2) cell-matrix interactions, and (3) fluid secretion [46]. Many factors can regulate these processes through different signaling pathways and are briefly described below.

(1) Intracellular cyclic adenosine monophosphate (cAMP) is likely the major driver of hepatic cyst growth [13]. In addition, cAMP levels are elevated in PCK rat cholangiocytes as compared to cholangiocytes from control, SD rats. Octreotide, a somatostatin analog, reduces hepatic and renal cyst expansion in PCK rats by decreasing cAMP levels [47]. Another factor that contributes to CWEC proliferation is low intracellular $\left[\mathrm{Ca}^{2+}\right]$, which is reduced in CWECs from PCK rat livers [48]. Activation of Trpv4, a calcium-permeable cation channel expressed in normal cholangiocytes, increases intracellular calcium levels and suppresses proliferation of cholangiocytes isolated from PCK rats in vitro [46]. Although the mechanisms of cyst growth have been well-described, whether targeting cAMP or intracellular $\left[\mathrm{Ca}^{2+}\right]$ will prevent disease progression in humans is inconsistent [13]. (2) Remodeling of extracellular matrix includes alteration of extracellular matrix composition, basement membrane thickness, and the activities of matrix metalloproteases (MMPs) and their inhibitors, all of which can lead to cyst expansion [49]. (3) In ARPKD, little is known about how fluid secretion impacts hepatic cyst expansion. Previous data suggest that cystic epithelia can respond to secretin and secrete fluid through activating cAMP-dependent signaling pathway $[50,51]$.

In contrast to the origin of cyst development in liver, renal cysts in ARPKD are commonly described as dilated collecting ducts [52]. In contrast to what is observed in ADPKD, dilated collecting ducts and distal tubules lined with cuboidal or columnar epithelia remain connected to the urinary system [53]. It remains unclear in ARPKD whether or not cysts with squamous epithelia cells detach from the tubular segment from which they are derived [54]. Cyst formation and expansion are associated with increased proliferation of renal epithelial cells [55] and altered fluid secretion [56]. Recent data suggest that cAMP induces renal epithelial cell proliferation and promotes cyst growth by activating PKA/BRaf/MAPK pathways in CWECs from ARPKD patients [57]. Similarly, renal epithelial cells also exhibit a lower level of intracellular $\left[\mathrm{Ca}^{2+}\right]$ and sustained reduction of intracellular $\left[\mathrm{Ca}^{2+}\right]$ in normal cells induces a cAMP-growth stimulated phenotype [57]. In addition, an increased level of epidermal growth factor (EGF) receptor is demonstrated in renal cyst fluid, which is consistent with an overexpression of EGF receptor (EGFR) mRNA and protein in renal epithelia in cpk mice [58]. The administration of EGFR tyrosine kinase inhibitor does not protect PCK rats from developing renal cysts, possibly due to an increased level of cAMP after treatment [59].

\section{Mechanisms of Fibrosis in ARPKD}

Liver fibrosis results from chronic liver injury in conjunction with the accumulation of extracellular matrix (ECM) proteins synthesized by myofibroblasts (MFB). In the liver, the major cell types that contribute to MFB formation are hepatic stellate cells (HSCs) and portal fibroblasts (PFs). Residing in the space of Disse, HSCs are the principal cell type responsible for collagen synthesis in response to liver injury or changes in ECM stiffness [60]. HSCs are also activated by various mediators released from Kupffer cells, the liverresident macrophage population, and include transforming growth factor-beta (TGF- $\beta$ ) and tumor necrosis factor-alpha (TNF- $\alpha$ ) [61]. In addition, produced by multiple cells types in liver, connective tissue growth factor (CTGF) promotes the activation of HSCs [62]. When HSCs are activated, they convert from quiescent cells into proliferative, fibrogenic, and contractile MFB and release a variety of inflammatory chemoattractants such as monocyte chemoattractant protein-1 (MCP-1) to recruit monocytes to the liver [63]. PFs are found in the portal tract area and play a predominant role in biliary fibrosis [64]. Although both cell types express alpha smooth muscle actin ( $\alpha \mathrm{SMA})$ upon activation, research suggests that the MFB population that 
contributes to CHF/ARPKD is likely derived from PFs [65]. Similar to HSCs, TGF- $\beta$ and CTGF are involved in the activation of PFs [66-68]. By contrast, TNF- $\alpha$ does not seem important for PF activation or fibrogenic potential [64]. In addition to their role in biliary fibrosis, activated portal MFB regulate cholangiocyte proliferation through activating $\mathrm{P} 2 \mathrm{Y}$ receptors on bile duct epithelia [69]. Following the activation of HSCs or PFs to MFB, two major events occur which promote fibrogenesis. First, activated MFB directly increase the synthesis and deposition of ECM proteins. Second, the MFB proliferate and amplify the fibrotic response [70].

Matrix degradation is an important mechanism to reverse fibrosis or cirrhosis and can restore normal liver architecture. Two kinds of matrix degradation mechanisms exist: "pathologic matrix degradation" that disrupts low density matrix and "restorative matrix degradation" that degrades excess scar [71]. Matrix remodeling is carried out through a fine balance between activities of MMPs and their inhibitors. MMPs are a family of enzymes secreted as proenzymes and are activated by proteolytic cleavage. They play a pivotal role in the regression of liver fibrosis by degrading ECM and inducing MFB apoptosis. Expression of tissue inhibitors of metalloproteinase 1 (TIMP-1) promotes fibrosis, first, by inhibiting MMP activity, and, second, by inhibiting MFB apoptosis [72].

The connection between cyst growth and fibrosis in $\mathrm{CHF} / \mathrm{ARPKD}$ is thus far unclear. Cystogenesis may be the initial event that disrupts normal ECM remodeling and induces fibrogenesis. This hypothesis is supported by the fact that HSCs and PFs are activated and differentiate into MFB when microenvironmental stiffness increases [66, 73]; enlarging cysts may promote this increase in mechanical stiffness and facilitate PF and HSC activation. Recent evidence suggests that bile duct epithelia directly regulate $\mathrm{PF}$ proliferation and $\mathrm{PF}$ transdifferentiation to $\mathrm{MFB}$ via release of MCP-1 [74]. These data indicate that release of MCP-1 is likely an additional link between cyst growth and fibrosis.

Compared to what has been established regarding development of hepatic fibrosis, little is known about renal fibrosis regarding the mechanisms and consequences in ARPKD. Although the patients exhibit very different renal symptoms, they always develop some degree of interstitial fibrosis accompanied with renal cysts [75]. In PCK rats, the renal interstitial fibrosis is not evident until 70 days of age, and the renal disease is more severe in males than in females [29].

\section{Mechanisms of Inflammation in ARPKD}

In addition to cyst growth and fibrosis, inflammation is another pathological feature of CHF/ARPKD. Although inflammation in the liver has not been well studied as that in the kidney, immune cells such as mast cells (MC) are found accumulated in the pericystic areas in livers of human CHF/ARPKD patients [76]. Consistently, we have observed pericystic MC infiltration in PCK rats (data not shown). Inhibition of MC degranulation and histamine release with cromolyn sodium, a MC stabilizer, decreases cholangiocyte proliferation in bile duct ligation-induced cholestasis
[77], suggesting MC may also contribute to cholangiocytederived, CWEC proliferation. Moreover, upregulation of genes involved in innate immune responses, including activated complement protein $3(\mathrm{C} 3 \mathrm{a})$ and the MCP-1 receptor, is detected in $c p k$ mice [78]. Taken together, these data suggest that activation of innate immune effector cells and associated proteins contributes to progression of $\mathrm{PKD}$ in general as well as in $\mathrm{CHF} / \mathrm{ARPKD}$.

Although PKD is not primarily considered an inflammatory disorder, accumulating evidence suggests that inflammation occurs in the early stage of the disease and may also drive disease progression. For example, macrophage infiltration is found in the renal interstitium in human ADPKD patients with kidney failure [79]. Activated macrophages stimulate vascular endothelial cell proliferation in vitro [80]. Further, TNF- $\alpha$, an inflammatory cytokine, is present in renal cyst fluid of human ADPKD and induces renal cyst formation through regulating polycystin-2 [81]. In ARPKD, M2-like macrophages are present in kidneys of patients and in $c p k$ mice [82], supporting a role of macrophages in the progression of ARPKD. Work from others has found increased MCP1 in kidneys [83] from PCK rats. Consistent with a role for MCP-1 and macrophage infiltration in progression in PKD, inhibition of MCP-1 synthesis with bindarit reduces renal inflammation and renal dysfunction but did not attenuate cyst growth [83]. Whether or not MCP-1 depletion affected hepatic inflammation, cyst growth, or fibrosis was not evaluated in this study.

We characterized hepatic cyst development in PCK rats by calculating liver/body weight ratio (\%) and quantifying cyst number, compared to SD rats from postnatal days (PND) 0 to 90 (Figure 1). Consistent with the work of others [29], our data suggest that PCK rats had increased liver/body weight ratios (Figure 1(a)) which was paralleled by an increase in the number of cysts from PND 10 onward (Figure 1(b)). Consistently, a positive correlation exists between liver weight and cyst number, further supporting a relationship between these disease-related parameters (Figure 1(c)). To characterize CWEC proliferation, we performed immunohistochemical staining for proliferating cell nuclear antigen (PCNA), a nuclear protein expressed in G1-M phase [84], in SD and PCK rat liver. In PCK rat liver, PCNA was strongly expressed in CWECs compared to limited PCNA-positive staining in cholangiocytes in SD rat liver (Figure 2(a)). Additional studies suggest that pericystic fibrosis occurs in parallel with biliary dysgenesis in PCK rats $[29,39]$. By using picrosirius red staining to localize ECM, we found an increased level of fibrillar collagen deposition in PCK rat liver compared to that observed in SD rats (Figure 2(b)). In addition to cyst growth and pericystic fibrosis in PCK rat liver, we recently measured hepatic MCP-1 transcript levels in SD and PCK rats as a surrogate marker of hepatic inflammation and driver of fibrosis. The expression of MCP-1 was elevated in PCK rat liver compared to SD rats at PND 5, 10, 20, and 30, which is consistent with the work showing that MCP-1 is upregulated in PCK rat kidney [83]. While further studies are required, these data suggest that increased inflammation, perhaps mediated by macrophages or PFs, is a potent contributor to CHF/ARPKD progression. 


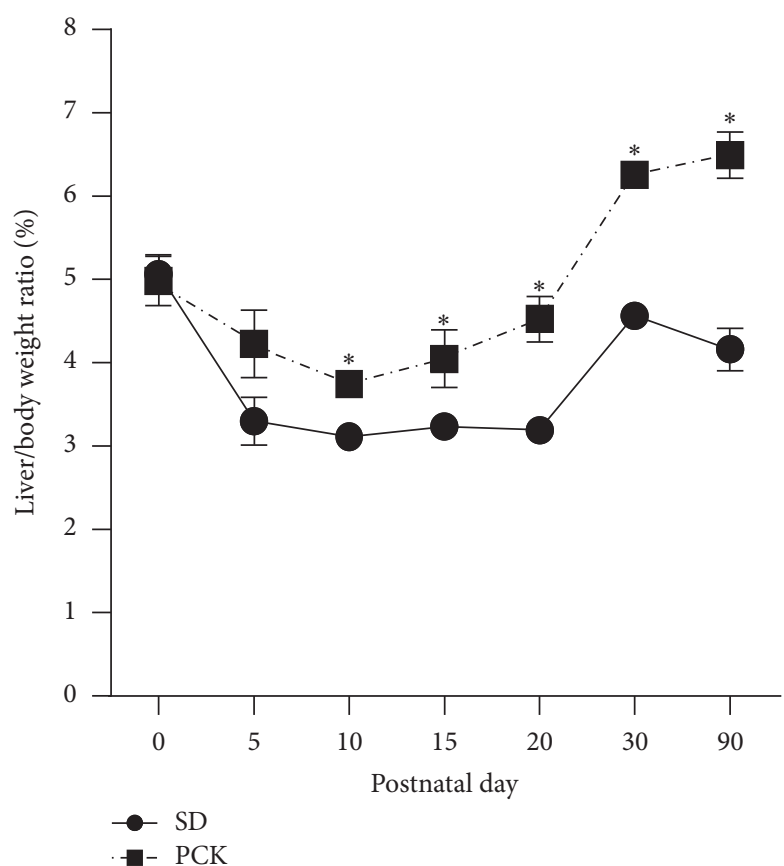

(a)

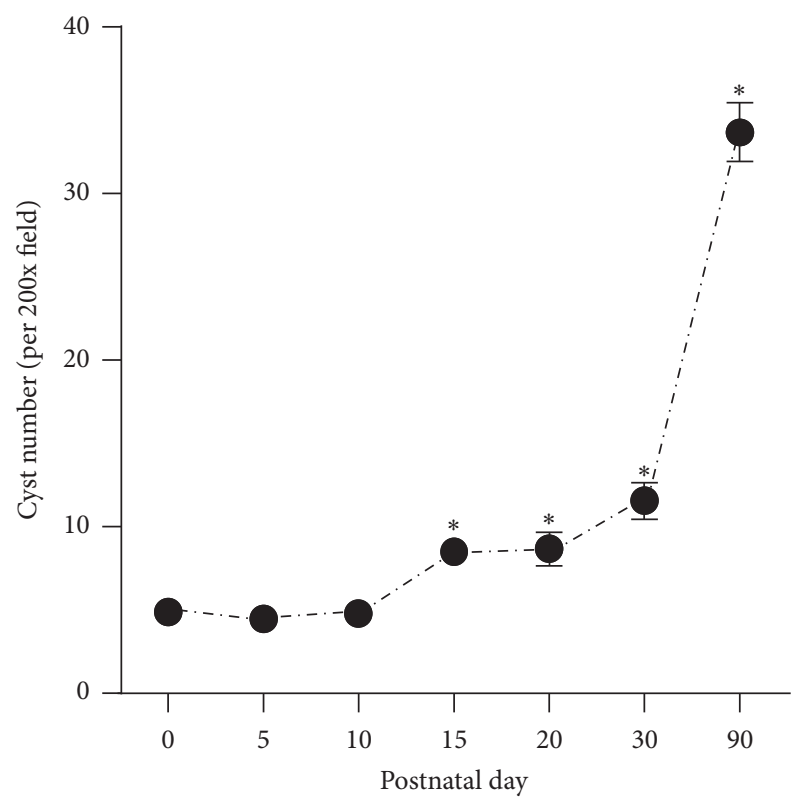

(b)

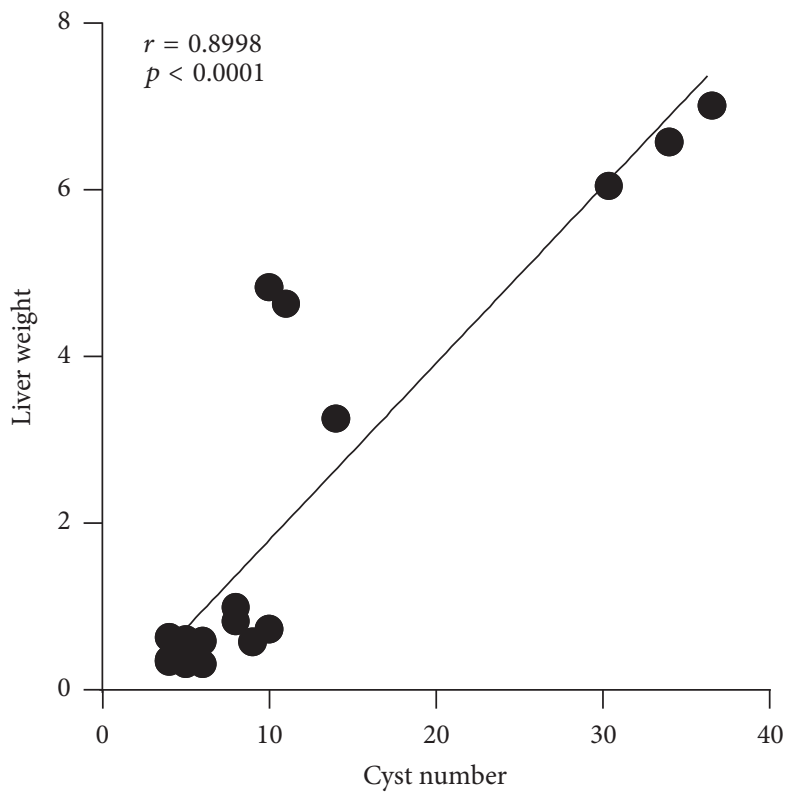

(c)

FIGURE 1: Characterization of hepatic cyst growth in PCK rats. (a) Liver/body weight ratio (as a percent of body weight) in Sprague-Dawley (SD) and polycystic kidney (PCK) rats was calculated from postnatal day (PND) 0 to PND 90. $*$, significantly different than SD rats at the indicated time point $(p<0.05)$. (b) Cyst number was quantified in 200x, hematoxylin and eosin-stained images from PCK rats between PND 0 and PND 90. $*$, significantly different than PND $0(p<0.05)$. (c) Pearson correlation of the relationship between liver weight and cyst number in PCK rats from PND 0 to PND 90. In all cases, $n=2-4$ rats per genotype per time point.

\section{8. "Pathogenic Triumvirate" in CHF/ARPKD: Insights into the Development of New Therapies}

Although mutations in the human $P K H D 1$ gene, or mutations in $P K H D 1$ orthologs in rats and mice, are required for development of CHF/ARPKD, other factors are also involved in disease progression. It is from a review of the published literature summarized in this paper that we propose a "pathogenic triumvirate" in CHF/ARPKD which includes three factors, cyst growth, fibrosis, and inflammation, as mediators which contribute to disease progression (Figure 3). 

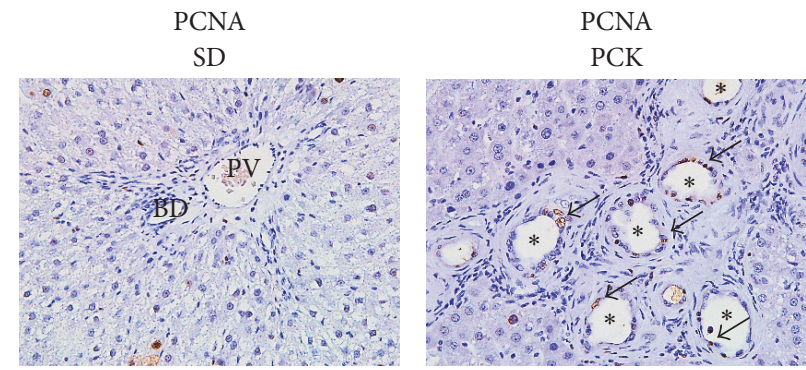

(a)
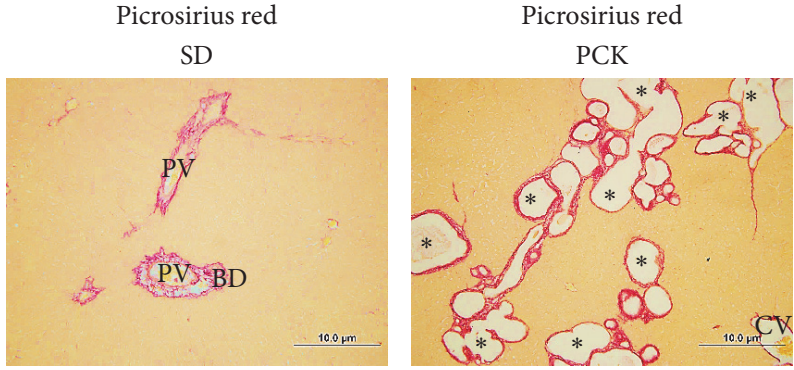

(b)

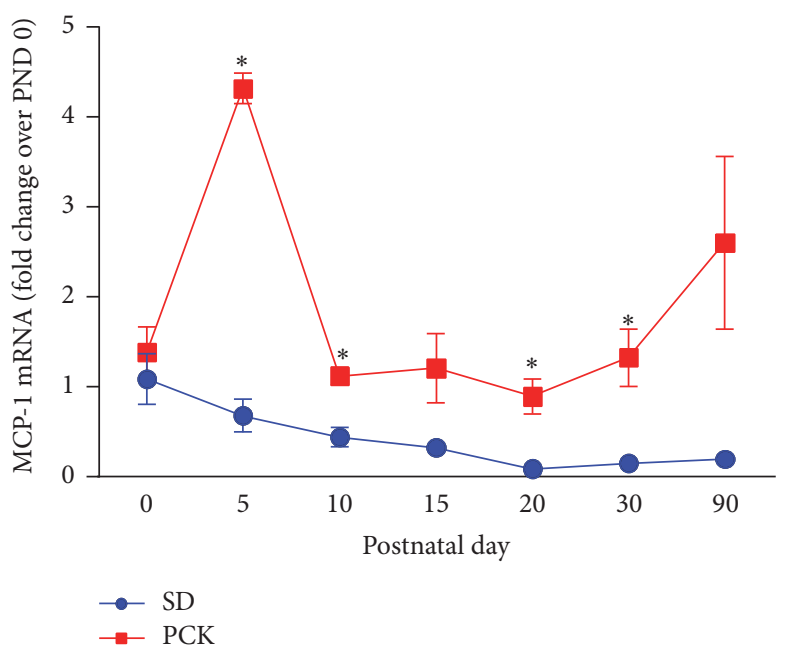

(c)

FIGURE 2: Cyst wall epithelial cell proliferation, fibrosis, and inflammation in PCK rats. (a) Hyperproliferation of cystic epithelia in polycystic kidney (PCK) rats. Hepatic PCNA content was assessed in Sprague-Dawley (SD) rats and PCK rats by immunohistochemistry. Images were taken at 400x magnification. Arrows indicate PCNA-positive cyst wall epithelial cells (CWECs) and asterisks indicate hepatic cysts. (b) Pericystic fibrosis in PCK rats. Extracellular matrix was localized in livers from SD and PCK rats by picrosirius red staining. Images were taken at 100x magnification. A scale bar $(100 \mu \mathrm{M})$ is included in each image. $\mathrm{PV}=$ portal vein, $\mathrm{CV}=$ central vein, and asterisks $=$ cysts $($ some, but not all are indicated). (c) Inflammation in PCK rats. Monocyte chemoattractant protein-1 (MCP-1) transcripts in SD (blue circles) and PCK (red squares) rats from postnatal days (PND) 0 to 90 were measured in liver using real-time PCR as a surrogate marker for inflammation. The data are expressed as fold change over control (SD) at PND 0. All images are representative of $n=2-4$ rats at each time point and data are graphed as means plus standard error of the mean. ${ }^{*} p<0.05$ between SD and PCK rats at the time points indicated.

Despite recent advances in our understanding of what contributes to the pathology of CHF/ARPKD, less is known about the molecular mechanisms regulating cyst growth, progression of fibrosis, and how inflammation contributes to these interrelated processes. Furthermore, whether or not a common mechanism drives members of the "pathogenic triumvirate" is also not known. We propose that leveraging what we do know about the CHF/ARPKD pathogenesis in the context of the pathogenic triumvirate will lead the way to new research and, possibly, new therapies for this disease. For example, finding a central mechanism that regulates all three components would be an attractive target for the development of new pharmacologic approaches to manage $\mathrm{CHF}$ / ARPKD. Alternatively, if a common mechanism does not exist, therapeutically targeting each member of the triumvirate concurrently may be a favorable approach.

\section{Summary and Conclusions}

$\mathrm{CHF} / \mathrm{ARPKD}$ is a genetic disease, but many factors contribute to its pathology and progression. Cyst growth is mainly regulated by cAMP and intracellular $\left[\mathrm{Ca}^{2+}\right]$ through stimulating cholangiocyte proliferation and fluid secretion. While these signals are clearly important in CHF/ARPKD, additional molecules and pathways which drive cell proliferation in cystic disease are being discovered and require further exploration. Development of hepatic fibrosis depends on the balance between ECM synthesis and degradation. Much research is needed not only to understand the role of the ECM in disease progression but also to define which cells are responsible for development of fibrosis in the first place (e.g., HSCs, PFs). Even less is known regarding the role inflammation plays in CHF/ARPKD. Future research should focus 


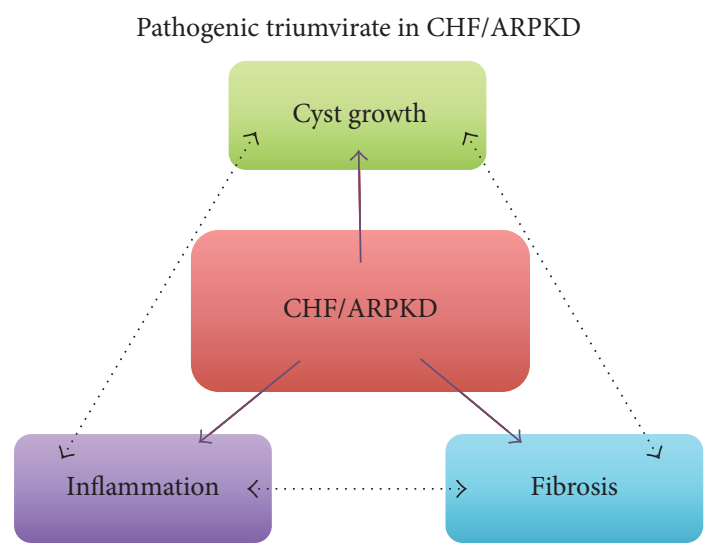

FIGURE 3: A "pathogenic triumvirate" in congenital hepatic fibrosis (CHF) in autosomal recessive polycystic kidney disease (ARPKD). Published data suggest that cyst growth, fibrosis, and inflammation drive CHF/ARPKD (solid purple arrows). We propose that relationships exist between cyst growth, fibrosis, and inflammation which drive progression of CHF/ARPKD (black, double-headed arrows with broken lines). Targeting a single pathway which drives each of the triumvirate members, or targeting multiple members concurrently, may provide better therapeutic strategies than if targeting any one member in isolation.

on identifying immune cell types and inflammatory mediators found in CHF/ARPKD and elucidating their roles in protection or promotion of disease. To date, the treatments for ARPKD are very limited and rely mostly on liver/kidney transplantation. Our "pathogenic triumvirate" identifies three target areas, cyst growth, fibrosis, and inflammation, which influence CHF/ARPKD progression. We believe that an integrated approach targeting each member of the pathogenic triumvirate at the same time, either by way of a common core pathway or three independent pathways, is required to improve therapeutic strategies for $\mathrm{CHF} / \mathrm{ARPKD}$. Further studies, utilizing the PCK rat or relevant mouse models of CHF/ARPKD, should strive to implement this idea in the preclinical arena.

\section{Competing Interests}

The authors declare that they have no competing interests.

\section{Acknowledgments}

This work was supported by grants from the University of Kansas Medical Center Kidney Institute (P30 DK106912), the University of Alabama at Birmingham Hepato-Renal Fibrocystic Disease Core Center (P30 DK074038), the National Center for Research Resources (P20 RR021940), the National Institute of General Medical Sciences (P20 GM103549), and the National Institute of Diabetes and Digestive and Kidney Diseases (R01 DK098414) of National Institutes of Health. Special thanks are due to the University of Kansas Medical Center Laboratory Animal Resource husbandry staff, veterinarians, the Office of Animal Welfare, and Institutional Animal Care and Use Committee for providing an excellent animal research program. Additional thanks go to Amukta Malyada Nuney for her editorial assistance.

\section{References}

[1] K. Zerres, G. Mücher, J. Becker et al., "Prenatal diagnosis of autosomal recessive polycystic kidney disease (ARPKD): molecular genetics, clinical experience, and fetal morphology," American Journal of Medical Genetics, vol. 76, no. 2, pp. 137-144, 1998.

[2] H. Blyth and B. G. Ockenden, "Polycystic disease of kidney and liver presenting in childhood," Journal of Medical Genetics, vol. 8, no. 3, pp. 257-284, 1971.

[3] V. Osathanondh and E. L. Potter, "Pathogenesis of polycystic kidneys. Type 1 due to hyperplasia of interstitial portions of collecting tubules," Archives of Pathology \& Laboratory Medicine, vol. 77, pp. 466-473, 1964.

[4] C. Bergmann, J. Senderek, E. Windelen et al., "Clinical consequences of $P K H D 1$ mutations in 164 patients with autosomalrecessive polycystic kidney disease (ARPKD)," Kidney International, vol. 67, no. 3, pp. 829-848, 2005.

[5] S. S. Williams, P. Cobo-Stark, L. R. James, S. Somlo, and P. Igarashi, "Kidney cysts, pancreatic cysts, and biliary disease in a mouse model of autosomal recessive polycystic kidney disease," Pediatric Nephrology, vol. 23, no. 5, pp. 733-741, 2008.

[6] J. Bernstein, M. Chandra, J. Creswell et al., "Renal-hepatic-pancreatic dysplasia: a syndrome reconsidered," American Journal of Medical Genetics, vol. 26, no. 2, pp. 391-403, 1987.

[7] B. L. Shneider and M. S. Magid, "Liver disease in autosomal recessive polycystic kidney disease," Pediatric Transplantation, vol. 9, no. 5, pp. 634-639, 2005.

[8] B. Turkbey, I. Ocak, K. Daryanani et al., "Autosomal recessive polycystic kidney disease and congenital hepatic fibrosis (ARPKD/CHF)," Pediatric Radiology, vol. 39, no. 2, pp. 100-111, 2009.

[9] L. Locatelli, M. Cadamuro, C. Spirlì et al., "Macrophage recruitment by fibrocystin-defective biliary epithelial cells promotes portal fibrosis in congenital hepatic fibrosis," Hepatology, vol. 63, no. 3, pp. 965-982, 2016.

[10] Y. Nakanuma, K. Harada, Y. Sato, and H. Ikeda, "Recent progress in the etiopathogenesis of pediatric biliary disease, particularly Caroli's disease with congenital hepatic fibrosis and biliary atresia," Histology and Histopathology, vol. 25, no. 2, pp. 223-235, 2010.

[11] J. Flores, R. Arnon, R. A. Morotti, L. Guay-Woodford, S. Emre, and B. L. Shneider, "Rapid evolution of congenital hepatic fibrosis after liver transplantation for acute liver failure: the potential role of extrahepatic factors," Liver Transplantation, vol. 14 , no. 5, pp. 660-664, 2008.

[12] T. F. Tracy Jr., P. Dillon, E. S. Fox, K. Minnick, and C. Vogler, "The inflammatory response in pediatric biliary disease: macrophage phenotype and distribution," Journal of Pediatric Surgery, vol. 31, no. 1, pp. 121-126, 1996.

[13] T. V. Masyuk, A. I. Masyuk, and N. F. La Russo, “Therapeutic targets in polycystic liver disease," Current Drug Targets, In press.

[14] J. M. Cobben, M. H. Breuning, C. Schoots, L. P. Ten Kate, and K. Zerres, "Congenital hepatic fibrosis in autosomal-dominant polycystic kidney disease," Kidney International, vol. 38, no. 5, pp. 880-885, 1990. 
[15] R. Salonen, "The Meckel syndrome: clinicopathological findings in 67 patients," American Journal of Medical Genetics, vol. 18, no. 4, pp. 671-689, 1984.

[16] M. Vankalakunti, K. Gupta, N. Kakkar, and A. Das, "Renalhepatic-pancreatic dysplasia syndrome (ivemark's syndrome)," Diagnostic Pathology, vol. 2, article 24, 2007.

[17] J. G. Gleeson, L. C. Keeler, M. A. Parisi et al., "Molar tooth sign of the midbrain-hindbrain junction: occurrence in multiple distinct syndromes," American Journal of Medical Genetics, vol. 125, no. 2, pp. 125-134, 2004.

[18] L. F. Onuchic, L. Furu, Y. Nagasawa et al., "PKHD1, the polycystic kidney and hepatic disease 1 gene, encodes a novel large protein containing multiple immunoglobulin-like plexin-transcription-factor domains and parallel beta-helix 1 repeats," American Journal of Human Genetics, vol. 70, no. 5, pp. 13051317, 2002.

[19] C. J. Ward, M. C. Hogan, S. Rossetti et al., "The gene mutated in autosomal recessive polycystic kidney disease encodes a large, receptor-like protein," Nature Genetics, vol. 30, no. 3, pp. 259269, 2002.

[20] L. F. C. Menezes, Y. Cai, Y. Nagasawa et al., "Polyductin, the PKHD1 gene product, comprises isoforms expressed in plasma membrane, primary cilium, and cytoplasm," Kidney International, vol. 66, no. 4, pp. 1345-1355, 2004.

[21] C. Bergmann, J. Senderek, B. Sedlacek et al., "Spectrum of mutations in the gene for autosomal recessive polycystic kidney disease (ARPKD/PKHD1)," Journal of the American Society of Nephrology, vol. 14, no. 1, pp. 76-89, 2003.

[22] P. C. Harris and S. Rossetti, "Molecular genetics of autosomal recessive polycystic kidney disease," Molecular Genetics and Metabolism, vol. 81, no. 2, pp. 75-85, 2004.

[23] L. M. Guay-Woodford and R. A. Desmond, "Autosomal recessive polycystic kidney disease: the clinical experience in North America," Pediatrics, vol. 111, no. 5, pp. 1072-1080, 2003.

[24] M. Goto, N. Hoxha, R. Osman, and K. M. Dell, “The reninangiotensin system and hypertension in autosomal recessive polycystic kidney disease," Pediatric Nephrology, vol. 25, no. 12, pp. 2449-2457, 2010.

[25] M. Gunay-Aygun, E. D. Avner, R. L. Bacallao et al., "Autosomal recessive polycystic kidney disease and congenital hepatic fibrosis: summary statement of a First National Institutes of Health/ Office of Rare Diseases conference," Journal of Pediatrics, vol. 149, no. 2, pp. 159-164, 2006.

[26] T. Sugiura, T. Endo, K. Ito et al., "Recurrent cholangitis with congenital hepatic fibrosis and pancreaticobiliary maljunction after Roux-en-Y reconstruction," European Journal of Pediatric Surgery Reports, vol. 1, no. 1, pp. 43-45, 2013.

[27] J. S. Ko, N.-J. Yi, K. S. Suh, and J. K. Seo, "Pediatric liver transplantation for fibropolycystic liver disease," Pediatric Transplantation, vol. 16, no. 2, pp. 195-200, 2012.

[28] M. Katsuyama, T. Masuyama, I. Komura, T. Hibino, and H. Takahashi, "Characterization of a novel polycystic kidney rat model with accompanying polycystic liver," Experimental Animals, vol. 49, no. 1, pp. 51-55, 2000.

[29] D. J. Lager, Q. Qian, R. J. Bengal, M. Ishibashi, and V. E. Torres, "The pck rat: a new model that resembles human autosomal dominant polycystic kidney and liver disease," Kidney International, vol. 59, no. 1, pp. 126-136, 2001.

[30] S. Nagao, M. Kugita, D. Yoshihara, and T. Yamaguchi, "Animal models for human polycystic kidney disease," Experimental Animals, vol. 61, no. 5, pp. 477-488, 2012.
[31] X. Y. Hou, M. Mrug, B. K. Yoder et al., "Cystin, a novel ciliaassociated protein, is disrupted in the cpk mouse model of polycystic kidney disease," The Journal of Clinical Investigation, vol. 109, no. 4, pp. 533-540, 2002.

[32] E. D. Avner, F. E. Studnicki, M. C. Young et al., "Congenital murine polycystic kidney disease-I. The ontogeny of tubular cyst formation," Pediatric Nephrology, vol. 1, no. 4, pp. 587-596, 1987.

[33] J. L. Ricker, V. H. Gattone II, J. P. Calvet, and C. A. Rankin, "Development of autosomal recessive polycystic kidney disease in BALB/c-cpk/cpk mice," Journal of the American Society of Nephrology, vol. 11, no. 10, pp. 1837-1847, 2000.

[34] V. H. Gattone II, J. P. Calvet, B. D. Cowley Jr. et al., "Autosomal recessive polycystic kidney disease in a murine model. A gross and microscopic description," Laboratory Investigation, vol. 59, no. 2, pp. 231-238, 1988.

[35] J. R. Woollard, R. Punyashtiti, S. Richardson et al., "A mouse model of autosomal recessive polycystic kidney disease with biliary duct and proximal tubule dilatation," Kidney International, vol. 72, no. 3, pp. 328-336, 2007.

[36] J. L. Bakeberg, R. Tammachote, J. R. Woollard et al., "Epitopetagged Pkhdl tracks the processing, secretion, and localization of fibrocystin," Journal of the American Society of Nephrology, vol. 22, no. 12, pp. 2266-2277, 2011.

[37] M. Moser, S. Matthiesen, J. Kirfel et al., "A mouse model for cystic biliary dysgenesis in autosomal recessive polycystic kidney disease (ARPKD)," Hepatology, vol. 41, no. 5, pp. 11131121, 2005.

[38] A.-R. Gallagher, E. L. Esquivel, T. S. Briere et al., "Biliary and pancreatic dysgenesis in mice harboring a mutation in Pkhdl," The American Journal of Pathology, vol. 172, no. 2, pp. 417-429, 2008.

[39] T. V. Masyuk, B. Q. Huang, A. I. Masyuk et al., "Biliary dysgenesis in the PCK rat, an orthologous model of autosomal recessive polycystic kidney disease," American Journal of Pathology, vol. 165, no. 5, pp. 1719-1730, 2004.

[40] A. I. Masyuk, T. V. Masyuk, and N. F. LaRusso, "Cholangiocyte primary cilia in liver health and disease," Developmental Dynamics, vol. 237, no. 8, pp. 2007-2012, 2008.

[41] D. Huangfu, A. Liu, A. S. Rakeman, N. S. Murcia, L. Niswander, and K. V. Anderson, "Hedgehog signalling in the mouse requires intraflagellar transport proteins," Nature, vol. 426, no. 6962, pp. 83-87, 2003.

[42] L. Schneider, C. A. Clement, S. C. Teilmann et al., "PDGFR $\alpha \alpha$ signaling is regulated through the primary cilium in fibroblasts," Current Biology, vol. 15, no. 20, pp. 1861-1866, 2005.

[43] M. Patterson, J. C. Gonzalez-Vitale, and C. J. Fagan, "Polycystic liver disease: a study of cyst fluid constituents," Hepatology, vol. 2, no. 4, pp. 475-478, 1982.

[44] M. J. Perugorria, T. V. Masyuk, J. J. Marin et al., "Polycystic liver diseases: advanced insights into the molecular mechanisms," Nature Reviews Gastroenterology and Hepatology, vol. 11, no. 12, pp. 750-761, 2014.

[45] E. S. Wills, R. Roepman, and J. P. H. Drenth, "Polycystic liver disease: ductal plate malformation and the primary cilium," Trends in Molecular Medicine, vol. 20, no. 5, pp. 261-270, 2014.

[46] S. A. Gradilone, T. V. Masyuk, B. Q. Huang et al., "Activation of Trpv4 reduces the hyperproliferative phenotype of cystic cholangiocytes from an animal model of ARPKD," Gastroenterology, vol. 139, no. 1, pp. 304-314.e2, 2010. 
[47] T. V. Masyuk, A. I. Masyuk, V. E. Torres, P. C. Harris, and N. F. Larusso, "Octreotide inhibits hepatic cystogenesis in a rodent model of polycystic liver disease by reducing cholangiocyte adenosine $3^{\prime}, 5^{\prime}$-cyclic monophosphate," Gastroenterology, vol. 132, no. 3, pp. 1104-1116, 2007.

[48] J. M. Banales, T. V. Masyuk, S. A. Gradilone, A. I. Masyuk, J. F. Medina, and N. F. LaRusso, "The cAMP effectors Epac and protein kinase a (PKA) are involved in the hepatic cystogenesis of an animal model of autosomal recessive polycystic kidney disease (ARPKD)," Hepatology, vol. 49, no. 1, pp. 160-174, 2009.

[49] P. D. Wilson, "Polycystic kidney disease," The New England Journal of Medicine, vol. 350, no. 2, pp. 151-164, 2004.

[50] G. T. Everson, M. Emmett, W. R. Brown, P. Redmond, and D. Thickman, "Functional similarities of hepatic cystic and biliary epithelium: studies of fluid constituents and in vivo secretion in response to secretin," Hepatology, vol. 11, no. 4, pp. 557-565, 1990.

[51] Q. Qian, "Isolated polycystic liver disease," Advances in Chronic Kidney Disease, vol. 17, no. 2, pp. 181-189, 2010.

[52] S. Kern, L.-B. Zimmerhackl, F. Hildebrandt, B. ErmischOmran, and M. Uhl, "Appearance of autosomal recessive polycystic kidney disease in magnetic resonance imaging and RARE-MR-urography," Pediatric Radiology, vol. 30, no. 3, pp. 156-160, 2000.

[53] C. Bergmann, "ARPKD and early manifestations of ADPKD: the original polycystic kidney disease and phenocopies," Pediatric Nephrology, vol. 30, no. 1, pp. 15-30, 2015.

[54] J. P. Calvet and J. J. Grantham, "The genetics and physiology of polycystic kidney disease," Seminars in Nephrology, vol. 21, no. 2, pp. 107-123, 2001.

[55] T. Nadasdy, Z. Laszik, G. Lajoie, K. E. Blick, D. E. Wheeler, and F. G. Silva, "Proliferative activity of cyst epithelium in human renal cystic diseases," Journal of the American Society of Nephrology, vol. 5, no. 7, pp. 1462-1468, 1995.

[56] E. I. Veizis, C. R. Carlin, and C. U. Cotton, "Decreased amiloride-sensitive $\mathrm{Na}^{+}$absorption in collecting duct principal cells isolated from BPK ARPKD mice," American Journal of Physiology-Renal Physiology, vol. 286, no. 2, pp. F244-F254, 2004.

[57] T. Yamaguchi, S. J. Hempson, G. A. Reif, A.-M. Hedge, and D. P. Wallace, "Calcium restores a normal proliferation phenotype in human polycystic kidney disease epithelial cells," Journal of the American Society of Nephrology, vol. 17, no. 1, pp. 178-187, 2006.

[58] S. Horikoshi, S. Kubota, G. R. Martin, Y. Yamada, and P. E. Klotman, "Epidermal growth factor (EGF) expression in the congenital polycystic mouse kidney," Kidney International, vol. 39, no. 1, pp. 57-62, 1991.

[59] V. E. Torres, W. E. Sweeney Jr., X. Wang et al., "Epidermal growth factor receptor tyrosine kinase inhibition is not protective in PCK rats," Kidney International, vol. 66, no. 5, pp. 1766-1773, 2004.

[60] E. Gäbele, D. A. Brenner, and R. A. Rippe, "Liver fibrosis: signals leading to the amplification of the fibrogenic hepatic stellate cell," Frontiers in Bioscience, vol. 8, pp. d69-d77, 2003.

[61] M. Matsuoka and H. Tsukamoto, "Stimulation of hepatic lipocyte collagen production by Kupffer cell-derived transforming growth factor $\beta$ : implication for a pathogenetic role in alcoholic liver fibrogenesis," Hepatology, vol. 11, no. 4, pp. 599605,1990 .

[62] G. Huang and D. R. Brigstock, "Regulation of hepatic stellate cells by connective tissue growth factor," Frontiers in Bioscience, vol. 17, no. 7, pp. 2495-2507, 2012.
[63] F. Marra, R. DeFranco, C. Grappone et al., "Increased expression of monocyte chemotactic protein-1 during active hepatic fibrogenesis: correlation with monocyte infiltration," The American Journal of Pathology, vol. 152, no. 2, pp. 423-430, 1998.

[64] J. A. Dranoff and R. G. Wells, "Portal fibroblasts: underappreciated mediators of biliary fibrosis," Hepatology, vol. 51, no. 4, pp. 1438-1444, 2010.

[65] J. Wen, "Congenital hepatic fibrosis in autosomal recessive polycystic kidney disease," Clinical and Translational Science, vol. 4, no. 6, pp. 460-465, 2011.

[66] Z. Li, J. A. Dranoff, E. P. Chan, M. Uemura, J. Sévigny, and R. G. Wells, "Transforming growth factor- $\beta$ and substrate stiffness regulate portal fibroblast activation in culture," Hepatology, vol. 46, no. 4, pp. 1246-1256, 2007.

[67] E. Borkham-Kamphorst, C. Schaffrath, E. Van de Leur et al., "The anti-fibrotic effects of CCN1/CYR61 in primary portal myofibroblasts are mediated through induction of reactive oxygen species resulting in cellular senescence, apoptosis and attenuated TGF- $\beta$ signaling," Biochimica et Biophysica ActaMolecular Cell Research, vol. 1843, no. 5, pp. 902-914, 2014.

[68] N. Sedlaczek, J.-D. Jia, M. Bauer et al., "Proliferating bile duct epithelial cells are a major source of connective tissue growth factor in rat biliary fibrosis," The American Journal of Pathology, vol. 158, no. 4, pp. 1239-1244, 2001.

[69] M. N. Jhandier, E. A. Kruglov, É. G. Lavoie, J. Sévigny, and J. A. Dranoff, "Portal fibroblasts regulate the proliferation of bile duct epithelia via expression of NTPDase2," The Journal of Biological Chemistry, vol. 280, no. 24, pp. 22986-22992, 2005.

[70] C. J. Parsons, M. Takashima, and R. A. Rippe, "Molecular mechanisms of hepatic fibrogenesis," Journal of Gastroenterology and Hepatology, vol. 22, no. 1, pp. S79-S84, 2007.

[71] S. L. Friedman, "Liver fibrosis-from bench to bedside," Journal of Hepatology, vol. 38, no. 1, pp. S38-S53, 2003.

[72] F. R. Murphy, R. Issa, X. Y. Zhou et al., "Inhibition of apoptosis of activated hepatic stellate cells by tissue inhibitor of metalloproteinase- 1 is mediated via effects on matrix metalloproteinase inhibition-implications for reversibility of liver fibrosis," The Journal of Biological Chemistry, vol. 277, no. 13, pp. 11069-11076, 2002.

[73] A. L. Olsen, S. A. Bloomer, E. P. Chan et al., "Hepatic stellate cells require a stiff environment for myofibroblastic differentiation," American Journal of Physiology - Gastrointestinal and Liver Physiology, vol. 301, no. 1, pp. G110-G118, 2011.

[74] E. A. Kruglov, R. A. Nathanson, T. Nguyen, and J. A. Dranoff, "Secretion of MCP-1/CCL2 by bile duct epithelia induces myofibroblastic transdifferentiation of portal fibroblasts," American Journal of Physiology-Gastrointestinal and Liver Physiology, vol. 290, no. 4, pp. G765-G771, 2006.

[75] F. E. Avni, G. Guissard, M. Hall, F. Janssen, V. DeMaertelaer, and F. Rypens, "Hereditary polycystic kidney diseases in children: changing sonographic patterns through childhood," Pediatric Radiology, vol. 32, no. 3, pp. 169-174, 2002.

[76] S. Ozaki, Y. Sato, M. Yasoshima, K. Harada, and Y. Nakanuma, "Diffuse expression of heparan sulfate proteoglycan and connective tissue growth factor in fibrous septa with many mast cells relate to unresolving hepatic fibrosis of congenital hepatic fibrosis," Liver International, vol. 25, no. 4, pp. 817-828, 2005.

[77] L. L. Kennedy, L. A. Hargrove, A. B. Graf et al., "Inhibition of mast cell-derived histamine secretion by cromolyn sodium treatment decreases biliary hyperplasia in cholestatic rodents," Laboratory Investigation, vol. 94, no. 12, pp. 1406-1418, 2014. 
[78] M. Mrug, J. Zhou, Y. Woo et al., "Overexpression of innate immune response genes in a model of recessive polycystic kidney disease," Kidney International, vol. 73, no. 1, pp. 63-76, 2008.

[79] M. Zeier, P. Fehrenbach, S. Geberth, K. Möhring, R. Waldherr, and E. Ritz, "Renal histology in polycystic kidney disease with incipient and advanced renal failure," Kidney International, vol. 42, no. 5, pp. 1259-1265, 1992.

[80] P. J. Polverini, R. S. Cotran, M. A. Gimbrone, and E. R. Unanue, "Activated macrophages induce vascular proliferation," Nature, vol. 269, no. 5631, pp. 804-806, 1977.

[81] X. Li, B. S. Magenheimer, S. Xia et al., "A tumor necrosis factor$\alpha$-mediated pathway promoting autosomal dominant polycystic kidney disease," Nature Medicine, vol. 14, no. 8, pp. 863-868, 2008.

[82] K. I. Swenson-Fields, C. J. Vivian, S. M. Salah et al., "Macrophages promote polycystic kidney disease progression," Kidney International, vol. 83, no. 5, pp. 855-864, 2013.

[83] C. Zoja, D. Corna, M. Locatelli et al., "Effects of MCP-1 inhibition by bindarit therapy in a rat model of polycystic kidney disease," Nephron, vol. 129, no. 1, pp. 52-61, 2015.

[84] N. H. Waseem and D. P. Lane, "Monoclonal antibody analysis of the proliferating cell nuclear antigen (PCNA). Structural conservation and the detection of a nucleolar form," Journal of Cell Science, vol. 96, no. 1, pp. 121-129, 1990. 


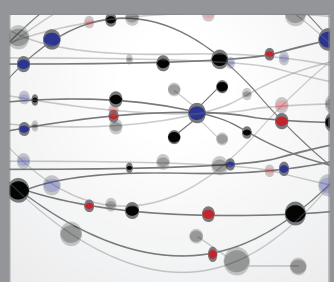

The Scientific World Journal
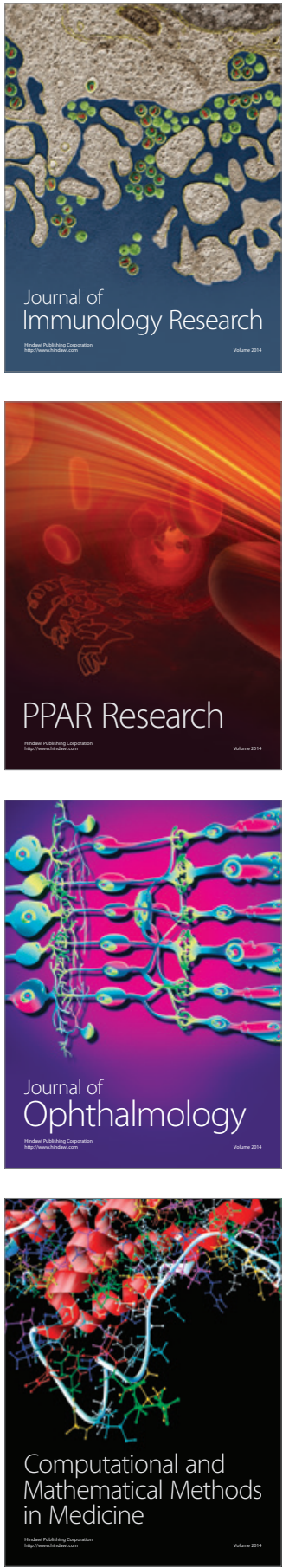

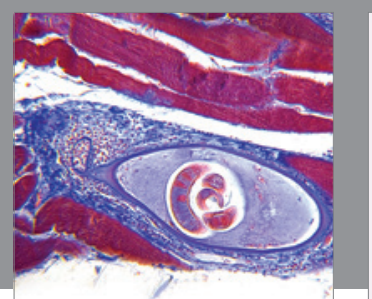

Gastroenterology Research and Practice

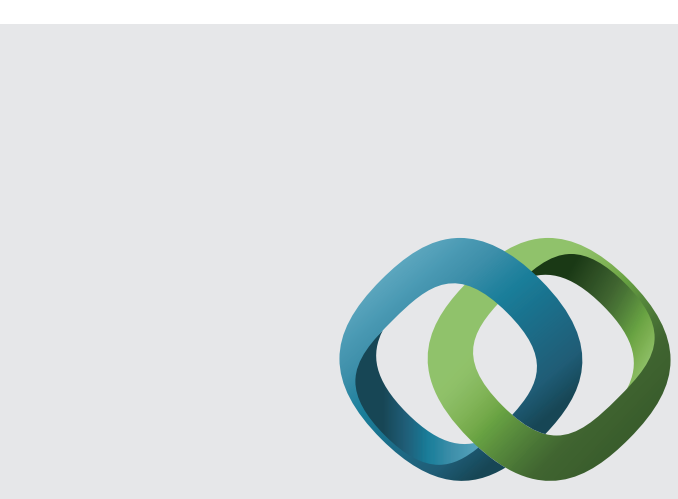

\section{Hindawi}

Submit your manuscripts at

http://www.hindawi.com
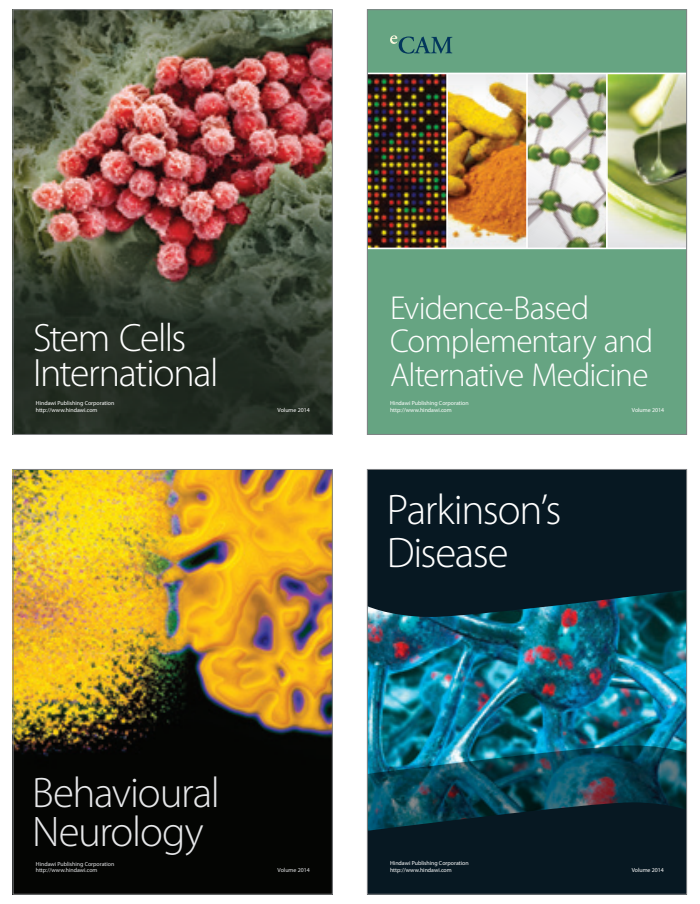
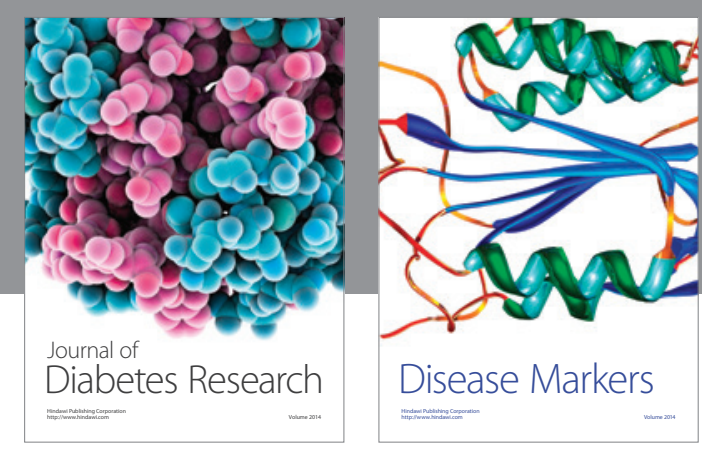

Disease Markers
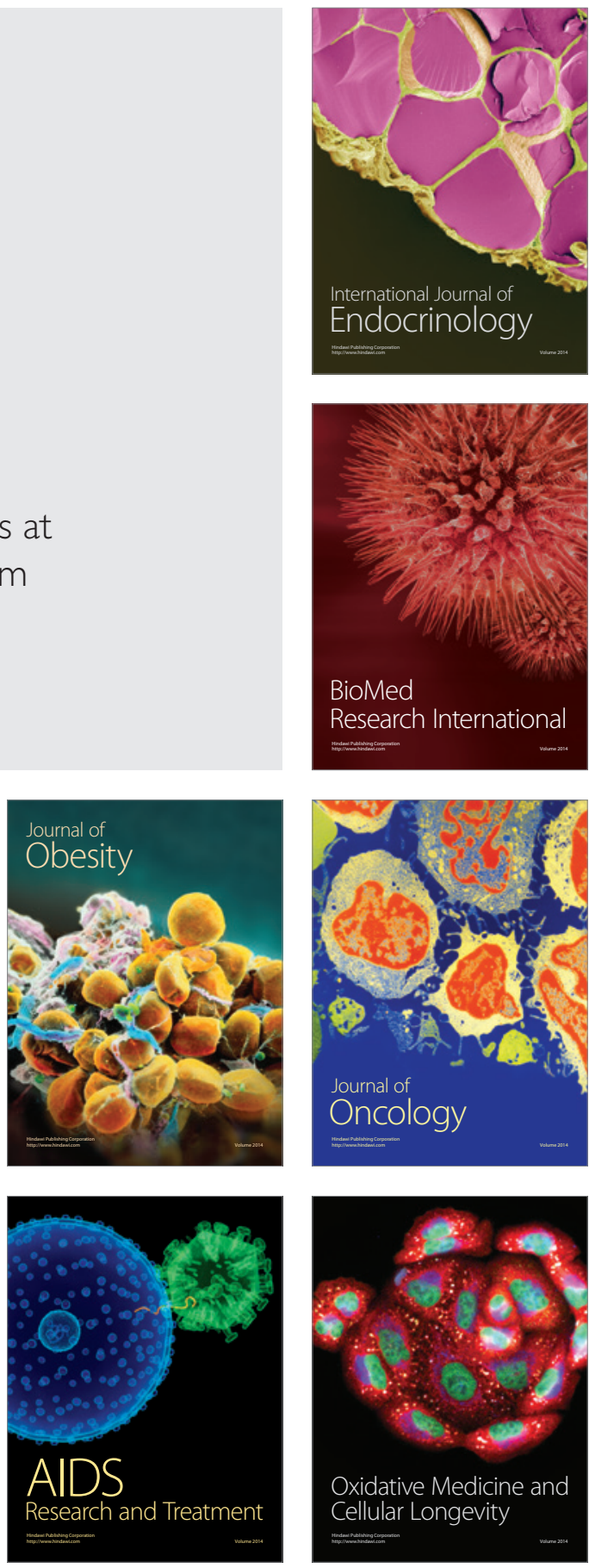